\title{
Integrated photonic platform based on semipolar InGaN/GaN multiple section laser diodes
}

\author{
Chao Shen ${ }^{1}$, Changmin Lee ${ }^{2}$, Tien Khee $\mathrm{Ng}^{1}$, James S. Speck², Shuji Nakamura², Steven P. DenBaars², and Boon S. \\ $\mathrm{Ooi}^{1 *}$ \\ ${ }^{1}$ Photonics Laboratory, King Abdullah University of Science and Technology (KAUST), Thuwal 23955, Saudi Arabia \\ ${ }^{2}$ Materials Department, University of California Santa Barbara (UCSB), Santa Barbara, CA 93106, USA \\ *E-mail address: boon.ooi@kaust.edu.sa
}

\begin{abstract}
The challenges to realizing III-nitride photonic integrated circuit (PIC) are discussed. Utilizing InGaN-based multi-section laser diode (LD) on semipolar GaN substrate, the seamless on-chip integration of III-nitride waveguide photodetector (WPD) in the visible regime has been demonstrated.

Keywords - diode laser; InGaN laser; visible lasers; photonic integration; waveguide photodetector; semipoalr; visible light communication
\end{abstract}

InGaN/GaN quantum well (QW) based optoelectronic devices are essential for light generation, transmission, modulation, and detection in the violet-blue-green color regime [1-6]. Recently, III-nitride laser diodes (LDs) have shown advantages as a viable high-power light source for a number of important applications, such as smart lighting, optical storage, free-space visible light communication, underwater wireless optical communication, and internet of things [1,7-9]. Currently, those functionalities have been demonstrated based on discrete devices, such as InGaN-based LDs, transverse-transmission modulators, and photodetectors [2, 3, 8, 9]. Though the on-chip integration of photonic devices in telecommunication wavelength has been demonstrated based on GaAs and InP material systems [10], such integrated photonic platform has not been realized in visible regime. The major challenge to achieving III-nitride photonic integrated circuit (PIC) utilizing conventional techniques is the large separation between the absorption and emission peak, i.e. the Stokes shift, in conventional c-plane orientated InGaN/GaN QWs. The simulation and experimental results reveal a large Stokes shift, originating from the large polarization field presented in QWs grown on polar, c-plane $\mathrm{GaN}$ or sapphire substrates. We furthermore propose, design, and demonstrate the integration of III-nitride photonic components based on the semipolar multisection LDs at $\sim 405 \mathrm{~nm}$. Benefiting from a significantly reduced polarization field, such photonic integration based on $\mathrm{GaN}$ material system can be achieved. For example, we demonstrated the integration of light-generation and modulation functionalities achieved by employing a small foot-print, low material polarization field integrated waveguide modulatorlaser diode (IWM-LD) [11]. configuration, fabricated seamlessly on semipolar GaN substrate. Utilizing an integrated absorber, a high optical power, droop-free, blue-emitting superluminescent diode (SLD) was demonstrated as a unique light-emitting component [12]. The fabrication and characterization of the high-performance waveguide photodetector (WPD) integrated with an LD sharing the single InGaN/GaN quantum well active region is presented [13]. The
WPD shows a large 3-dB modulation bandwidth of $230 \mathrm{MHz}$. The integrated device, being used for power monitoring and onchip communication, offers the advantages of small-footprint, high-speed, and low power consumption. The findings are significant in paving the way towards the eventual realization of III-nitride on-chip photonic system.

\section{ACKNOWLEDGMENT}

We acknowledge the financial support from King Abdulaziz City for Science and Technology (KACST), Grant No. KACST TIC R2-FP-008, and KACST-KAUST-UCSB solid state lighting program (SSLP). This work is partially supported by baseline funding, BAS/1/1614-01-01, from the King Abdullah University of Science and Technology (KAUST).

\section{REFERENCES}

[1] S. Pimputkar, J. S. Speck, S. P. DenBaars, and S. Nakamura, "Prospects for LED lighting," Nature Photonics, 3, 180-182 (2009).

[2] C. K. Kao, A. Bhattacharyya, C. Thomidis, R. Paiella, and T. D. Moustakas, "Electroabsorption modulators based on bulk GaN films and GaN/AlGaN multiple quantum wells," Journal of Applied Physics, 109, 083102 (2011)

[3] B. Butun, T. Tut, E. Ulker, T. Yelboga, and E. Ozbay, "High-performance visible-blind GaN-based p-i-n photodetectors," Applied Physics Letters, 92, 033507 (2008)

[4] C. Shen, T. K. Ng, and B. S. Ooi, "Enabling area-selective potentialenergy engineering in $\mathrm{InGaN} / \mathrm{GaN}$ quantum wells by post-growth intermixing," Optics Express, 23, 7991-7998 (2015).

[5] C. Zhao, T. K. Ng, N. Wei, A. Prabaswara, M. S. Alias, B. Janjua, et al., "Facile formation of high-quality $\mathrm{InGaN} / \mathrm{GaN}$ quantum-disks-innanowires on bulk-metal substrates for high-power light-emitters," Nano Letters, 16, 1056-1063 (2016).

[6] C. Shen, T. K. Ng, Y. Yang, D. Cha, and B. S. Ooi, "InGaN micro-LEDpillar as the building block for high brightness emitters," 2013 IEEE Photonics Conference (IPC), 174-175 (2013).

[7] C. Lee, C. Shen, H. M. Oubei, M. Cantore, B. Janjua, T. K. Ng, et al., "2 Gbit/s data transmission from an unfiltered laser-based phosphorconverted white lighting communication system," Optics Express, 23, 29779-29787 (2015)

[8] C. Shen, Y. Guo, H. M. Oubei, T. K. Ng, G. Liu, K.-H. Park, et al., "20meter underwater wireless optical communication link with $1.5 \mathrm{Gbps}$ data rate," Optics Express, 24, 25502-25509 (2016).

[9] M. T. Hardy, D. F. Feezell, S. P. DenBaars, and S. Nakamura, "Group IIInitride lasers: a materials perspective," Materials Today, 14, 408-415 (2011).

[10] L. A. Coldren, S. C. Nicholes, L. Johansson, S. Ristic, R. S. Guzzon, E. J. Norberg, et al., "High Performance InP-Based Photonic ICs-A Tutorial," Journal of Lightwave Technology, 29, 554-570 (2011). 
[11] C. Shen, T. K. Ng, J. T. Leonard, A. Pourhashemi, H. M. Oubei, M. S. Alias, et al., "High-modulation-efficiency, integrated waveguide modulator-laser diode at $448 \mathrm{~nm}$," ACS Photonics, 3, 262-268 (2016).

[12] C. Shen, T. K. Ng, J. T. Leonard, A. Pourhashemi, S. Nakamura, S. P. DenBaars, et al., "High-brightness semipolar (2021) blue InGaN/GaN superluminescent diodes for droop-free solid-state lighting and visiblelight communications," Optics Letters, 41, 2608-2611 (2016).

[13] C. Shen, C. Lee, E. Stegenburgs, J. H. Lerma, T. K. Ng, S. Nakamura, et al., "Semipolar III-nitride quantum well waveguide photodetector integrated with laser diode for on-chip photonic system," Applied Physics Express, 10, 042201 (2017). 